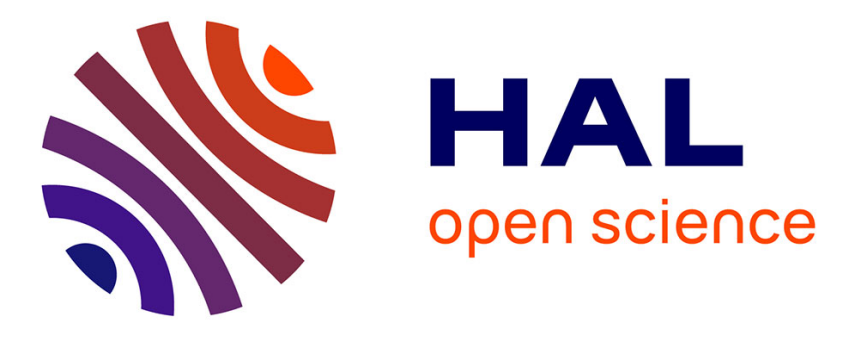

\title{
Subcriticality of the zigzag transition: A nonlinear bifurcation analysis
}

Tommy Dessup, Christophe Coste, Michel Saint-Jean

\section{To cite this version:}

Tommy Dessup, Christophe Coste, Michel Saint-Jean. Subcriticality of the zigzag transition: A nonlinear bifurcation analysis. Physical Review E: Statistical, Nonlinear, and Soft Matter Physics, 2015, 91, pp.32917 - 32917. 10.1103/PhysRevE.91.032917 . hal-01404811

\section{HAL Id: hal-01404811 \\ https://hal-univ-paris.archives-ouvertes.fr/hal-01404811}

Submitted on 29 Nov 2016

HAL is a multi-disciplinary open access archive for the deposit and dissemination of scientific research documents, whether they are published or not. The documents may come from teaching and research institutions in France or abroad, or from public or private research centers.
L'archive ouverte pluridisciplinaire HAL, est destinée au dépôt et à la diffusion de documents scientifiques de niveau recherche, publiés ou non, émanant des établissements d'enseignement et de recherche français ou étrangers, des laboratoires publics ou privés. 


\title{
Subcriticality of the zigzag transition: A nonlinear bifurcation analysis
}

\author{
Tommy Dessup, Christophe Coste, and Michel Saint Jean \\ Laboratoire “Matière et Systèmes Complexes" (MSC), UMR 7057 CNRS, Université Paris 7 Diderot, 75205 Paris Cedex 13, France
}

(Received 2 July 2014; published 20 March 2015)

\begin{abstract}
When repelling particles are confined by a transverse potential in quasi-one-dimensional geometry, the straight line equilibrium configuration becomes unstable at small confinement, in favor of a staggered row that may be inhomogeneous or homogeneous. This conformational phase transition is a pitchfork bifurcation called the zigzag transition. We study the zigzag transition in infinite and periodic finite systems with short-range interactions. We provide numerical evidence that in this case the bifurcation is subcritical since it exhibits phase coexistence and hysteretic behavior. The physical mechanism responsible for the change in the bifurcation character is the nonlinear coupling between the transverse soft mode at the transition and the longitudinal Goldstone mode linked to the translational or rotational invariance of the zigzag pattern. An asymptotic analysis, near the bifurcation threshold and assuming an infinite system, gives an explicit expression for the normal form of the bifurcation. We establish the subcriticality, and we describe with excellent precision the inhomogeneous zigzag patterns observed in the simulations. A direct test of the physical mechanism responsible for the bifurcation character evidences a quantitative agreement.
\end{abstract}

DOI: 10.1103/PhysRevE.91.032917

PACS number(s): 05.45.-a, 02.30.Oz, 47.20.Hw, 47.20.Ky

\section{INTRODUCTION}

The zigzag transition is a configurational phase transition that happens when repelling particles in a plane are confined in quasi-one-dimensional (quasi-1D) linear traps by a transverse potential. When the transverse confinement potential is much greater than the interparticle energy, all particles are aligned along the trap axis at equilibrium. Below a critical transverse confinement, the equilibrium pattern undergoes a transition from a straight line toward a staggered row (homogeneous or inhomogeneous), which is called "zigzag."

The zigzag transition is easily described if one assumes a perfect zigzag pattern. Let us consider identical point particles of mass $m$ located in the plane $(x O y)$, transversally confined in a quasi-1D geometry by a harmonic potential of stiffness $\beta$, and interacting with a repulsive potential $U(r)$. Here $O x$ is the longitudinal axis of the cell and $O y$ is the transverse axis. In an infinite system (or with periodic boundary conditions in the longitudinal direction and an even number of particles), the zigzag pattern corresponds to particles located at points $A_{i}$ of coordinates $\{2 i d,-h\}$ and at points $B_{i}$ of coordinates $\{(2 i+1) d,+h\}$, where the zigzag height $h$ is a constant (see Fig. 1).

The potential energy of any particle in Fig. 1, up to secondneighbor interactions, is

$$
\begin{aligned}
E= & U\left[\sqrt{d^{2}+4 h^{2}}\right]+U(2 d)+\frac{\beta}{2} h^{2} \\
\approx & U(d)+U(2 d)+h^{2}\left[\frac{\beta}{2}+\frac{2 U^{\prime}(d)}{d}\right] \\
& +\frac{2 h^{4}}{d^{3}}\left[d U^{\prime \prime}(d)-U^{\prime}(d)\right],
\end{aligned}
$$

where the rightmost member results from an expansion in $h$ up to $h^{4}$. This energy is invariant toward the axial symmetry $y \longrightarrow-y$. When we decrease $\beta$, the minimum of energy at $h=0$ becomes a local maximum, with two symmetric minima $\pm h$ with $h>0$. The conformational transition takes place at a critical value $\beta_{Z Z}=-4 U^{\prime}(d) / d$. The energy landscape is shown in Fig. 2 for $\beta<\beta_{Z Z}$. It exhibits the characteristic features of a supercritical pitchfork bifurcation.

The zigzag transition has been observed with ions in Paul's trap [1-7], with plasma dusts [8-12], with colloids [13], and with millimetric charged beads [14-16]. A supercritical bifurcation has been observed in simulations of periodic systems with Coulomb interaction [17-20] and also in some periodic systems with short-range interactions [14,21]. Most experiments have been done in linear traps of finite length, with repulsive boundary conditions at the edges. In these traps, the particles are not equidistant, and the simple expression (1) is not strictly valid. The equilibrium pattern is spindleshaped, with the maximum zigzag height in the trap center. Nevertheless, one may expect the same phenomenology, with the uniform zigzag height $h$ replaced by the maximum height. In these finite systems, the bifurcation is always found to be supercritical, for Coulomb systems $[3,4,7,18]$ as well as for systems with short-range interactions [11,13-15,22].

In contrast, other experimental evidence contradicts the supercritical character of the bifurcation in periodic systems with short-range interactions. With plasma dust confined in an annulus, Sheridan [10] has observed a stable localized zigzag pattern, surrounded by aligned particles. We have also reported observations of inhomogeneous patterns and demonstrated that, for short-range interactions, the zigzag pattern in a periodic system is linearly unstable [16]. A subcritical bifurcation diagram has also been exhibited in the experiments of Straube et al. [13].

We can infer from the known literature that the character of the zigzag bifurcation in periodic systems is not necessarily the same as in finite systems with repulsive boundary conditions at the edges. This phenomenon has already been observed in the framework of instabilities, such as convection rolls [23-26], surface waves [27], or solidification fronts [28-30] in extended or annular geometries. In all of these systems, a supercritical pitchfork bifurcation was expected, while experiments in extended periodic cells exhibited a subcritical behavior. Because of the translational or rotational invariance of the system, the nonlinearities induce a coupling between the soft 


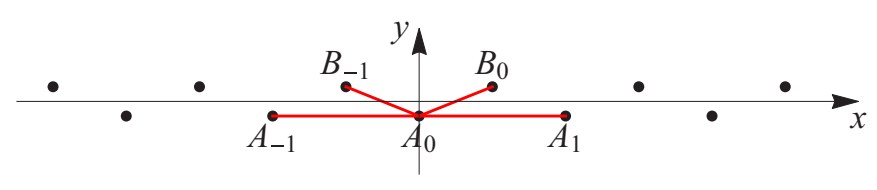

FIG. 1. (Color online) A schematic illustration of the zigzag pattern. The solid lines indicate the interactions that are taken into account in the theoretical description.

mode due to the instability and the Goldstone mode due to the continuous symmetry breaking and therefore the subcriticality of the bifurcation [31-33].

In Ref. [32], the terms of the normal form of the bifurcation are deduced from symmetry arguments, but with numerical coefficients that are not explicitly given by the analysis. In our approach, the underlying microscopic dynamics of the zigzag transition is known from first principles, and the normal form is obtained in a constructive way. The normal form fulfills all the required symmetry properties, and it exhibits the subcritical character of the bifurcation. Moreover, explicit expressions are given for all coefficients of the normal form, which allow a quantitative comparison between the observed inhomogeneous zigzag patterns and the calculations based on the normal form. The coupling between the soft mode at the bifurcation and the phase mode due to the breaking of a continuous symmetry induces a shift of the longitudinal interparticle distance. We are able to test quantitatively this effect, and we find an excellent agreement with our simulations, validating with great precision the mechanism that traces back to the coupling between the two soft modes.

This paper is organized as follows: Section II reports on numerical simulations and provides evidence of a subcritical zigzag transition in periodic systems with short-range interactions. In Sec. III, we construct perturbatively the normal form near the bifurcation in the thermodynamic limit. We identify the range in the bifurcation parameter for which inhomogeneous zigzag patterns exist, and we calculate the shapes of these patterns. In Sec. IV, we take into account the finite-size effects and compare quantitatively the inhomogeneous zigzag patterns observed in our simulations to the calculated patterns. A conclusion in Sec. V summarizes our findings, and four Appendixes are devoted to some technicalities.

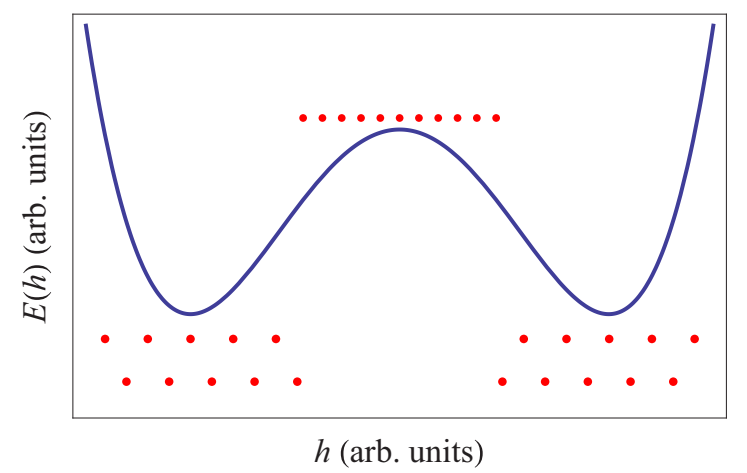

FIG. 2. (Color online) Schematic plot of the energy $E(h)$ as a function of the zigzag height $h$, for $\beta<\beta_{Z Z}$, with a sketch of the relevant patterns [see Eq. (1)].

\section{EVIDENCE OF A SUBCRITICAL ZIGZAG BIFURCATION}

\section{A. Equilibrium configurations}

To discuss the equilibrium configurations, it is convenient to introduce the distance to threshold $\epsilon$ as $\beta=\beta_{Z Z}(1-\epsilon)$. In Fig. 3(a), we plot the maximum height $h$ of the observed zigzag pattern as a function of $\epsilon$ and compare it to the height $h^{*}$ that minimizes the interaction energy (1) and corresponds to the perfect zigzag. Three areas may be identified in this plot. Near the threshold $\epsilon=0, h$ is small, scales as $\epsilon^{1 / 2}$, and is accurately given by the energy minimization [see Fig. 3(b)]. Increasing $\epsilon$, the equilibrium pattern becomes inhomogeneous, as shown by the insets of Fig. 3(a), and the maximum zigzag height is larger than for a perfect zigzag pattern: $h>h^{*}$. Then when $\epsilon$ is further increased, a high height zigzag pattern is recovered, of height $h^{*}$. The extension of the interval of $\epsilon$ values for which inhomogeneous patterns are observed increases with the system size (at constant density and interaction range).

\section{B. Linear instability of the zigzag pattern}

A linear stability analysis of the zigzag pattern has been done in Ref. [16]. In the zigzag lattice, the longitudinal and transverse vibrations both exhibit high-frequency optical branches and low-frequency acoustical branches. For finite range interactions, we have shown that, in an infinite system, there is a whole band of small wave numbers for which transverse acoustic vibrations have a pure imaginary frequency, $\omega_{\mathrm{AT}}$. The relevant parameters are the zigzag height $h$ and the interaction range $\lambda_{0}$, expressed in units of the mean interparticle distance $d$. For small enough $\lambda_{0} / d$ and small enough wave number, we have exhibited a range in zigzag height $h_{C 1} \leqslant h \leqslant h_{C 2}$ for which the frequency $\omega_{\mathrm{AT}}$ is pure imaginary. These limiting heights are shown in Ref. [16] to bound the area of existence of the inhomogeneous patterns (see the right plot of Fig. 3). The appearance of inhomogeneous patterns is thus linked to a long-wavelength instability, which explains why they are more easily observed in large systems.

The heights $h_{C 1}$ and $h_{C 2}$ depend on the wave number $q$, with $h_{C 1} \propto q$ when $q \rightarrow 0$. In a finite periodic system, the minimum nonzero wave number $q_{\min }$ scales as the inverse of the system size $L$, thus $h_{C 1} \propto 1 / L$. Near the transition, the zigzag height scales as $\epsilon^{1 / 2}$, where $\epsilon$ is the distance to threshold. There is therefore a range in $\epsilon$ that scales as $1 / L^{2}$ for which the bifurcation is supercritical. This explains the supercritical behavior evidenced by Fig. 3(b) and our previous results in Ref. [14].

\section{Hysteretic behavior}

In Fig. 4, we plot the maximum zigzag height $h$ as a function of the ratio $\beta / \beta_{Z Z}$, for increasing and decreasing stiffnesses, and several system sizes. The density and interaction potential are kept constant. The evolution of the zigzag height clearly exhibits an hysteretic behavior. For decreasing confining transverse stiffness $\beta$, the bifurcation between a straight line $h=0$ and a state with localized bifurcated patterns always happens at the same value, $\beta_{\text {down }}$. When $\beta$ increases, the transition toward the state $h=0$ takes place at a value 

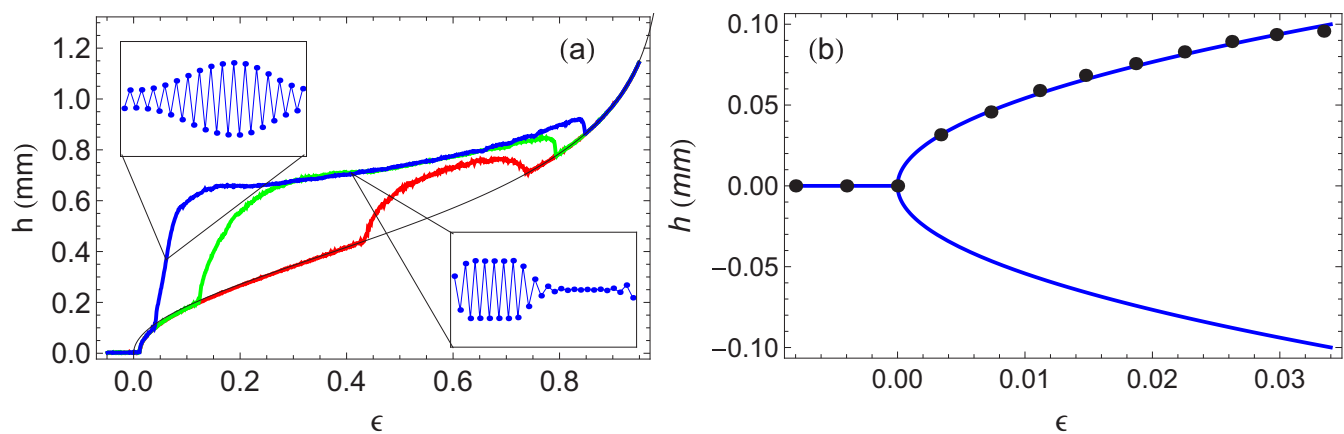

FIG. 3. (Color online) (a) Zigzag height $h(\mathrm{~mm})$ as a function of $\epsilon$ for 8 (thick red curve), 16 (thick green curve), and 32 (thick blue curve) particles. The fine solid black line indicates the expected height for a zigzag pattern $h^{*}$. For inhomogeneous zigzag, $h$ is the maximum height of the pattern. In the insets, we show two typical inhomogeneous patterns. The dots are simulations data, while the lines are only guide to the eyes. (b) Onset of the zigzag transition zoomed near the bifurcation threshold for 32 particles. The solid line is the theoretical height $h^{*}$ of a zigzag pattern, while the dots are simulations data.

$\beta_{\text {up }}>\beta_{\text {down }}$, and the greater the system size, the greater the difference $\beta_{\text {up }}-\beta_{\text {down }}>0$.

In Fig. 5, we plot the evolution of $\beta_{\text {down }} / \beta_{Z Z}$ and $\beta_{\text {up }} / \beta_{Z Z}$ with the particles number $N$. This confirms that $\beta_{\text {down }}$ is basically independent of $N$. In contrast, $\beta_{\text {up }}$ increases and tends toward a limit value $\beta_{\text {up }}^{\infty}$ with $\beta_{\text {up }}^{\infty}-\beta_{\text {up }}^{N} \propto 1 / N$. We have done these measurements at two temperatures. There is indeed no significant differences between the two sets of data, which ensures that we actually observe equilibrium patterns.

\section{Discussion}

Under the assumption of a perfect zigzag pattern, with equidistant particles alternately displaced from the bottom of the transverse potential well, the zigzag transition is found to be a supercritical pitchfork bifurcation. However, when an even number of particles is placed in a periodic cell, the particle distribution at equilibrium may be inhomogeneous. Moreover, we have shown in [16] that the vibration modes of the zigzag pattern exhibit a band of unstable acoustic modes.

The observation of inhomogeneous patterns evidences the coexistence between bifurcated $(h \neq 0)$ and nonbifurcated $(h=0)$ regions, which in the framework of Landau phase theory takes place for first-order phase transitions and not for second-order ones. Retranscribed in the language of bifurcations, this evidences a subcritical behavior of the pitchfork bifurcation for large systems. The subcritical character of the bifurcation is also evidenced by the hysteretic evolution of the maximum height of the equilibrium patterns when the transverse confinement is varied. The domain of stability of the inhomogeneous patterns (see Fig. 3) and the amplitude of the hysteresis cycles (see Figs. 4 and 5) depend on the
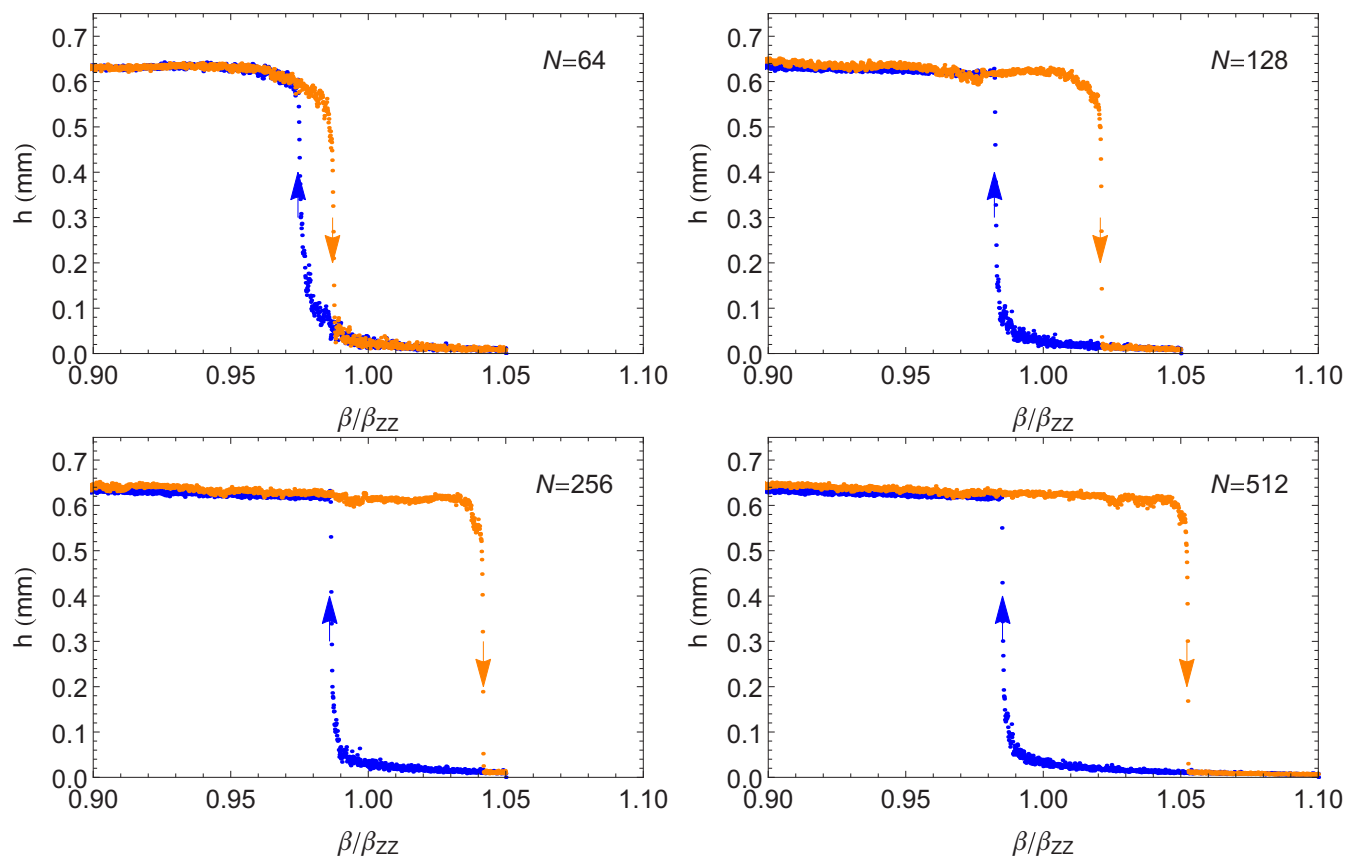

FIG. 4. (Color online) Evolution of the zigzag height $h(\mathrm{~mm})$ for increasing (orange points, see arrows) and decreasing (blue points, see arrows) $\beta / \beta_{Z Z}$. The relevant particle number $N$ is indicated in the upper right corner of each plot. The mean interparticle distance $d$ is the same in all plots. 


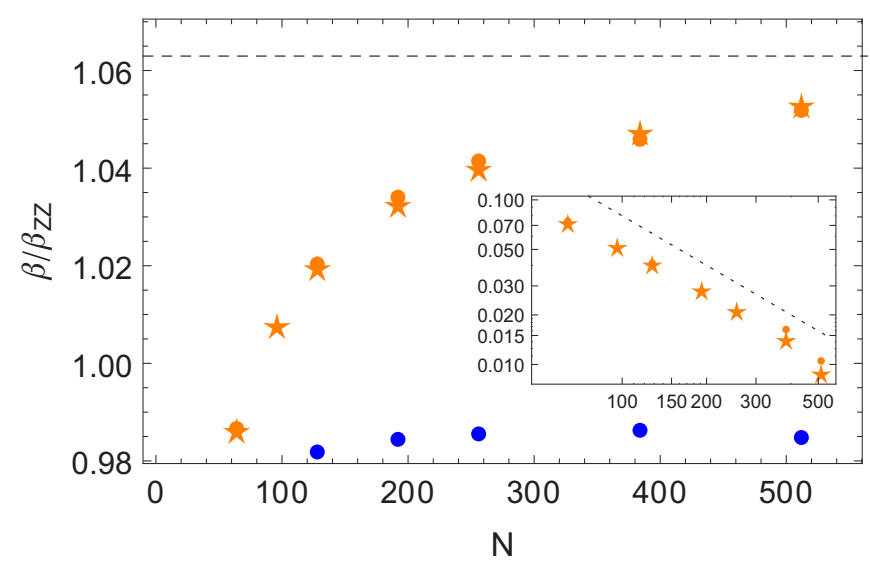

FIG. 5. (Color online) Reduced stiffness $\beta / \beta_{Z Z}$ at which a transition between aligned particles and localized zigzag happens as a function of the particle number $N$, at constant $d$. Blue dots are the measured values $\beta_{\text {down }}$ for decreasing $\beta$. Orange dots and stars are the measured values $\beta_{\text {up }}$ for increasing $\beta$. The thermodynamic temperature in the simulations (see Appendix A) is such that $U(d) / k_{B} T_{b}=10^{-2}$ (dots) and $U(d) / k_{B} T_{b}=10^{-4}$ (stars). The dashed line is the maximum height of the hysteresis calculated from the normal form [see Eq. (11)]. The inset is a log-log plot of the deviation to this maximum height as a function of $N$. The dotted line is of slope -1 .

system size, which implies that finite-size effects have to be taken into account.

The key ingredients for subcriticality, as will be shown in Sec. III, are the invariance of the system with a continuous symmetry, either translational or rotational, and the short range of the interactions. Using techniques developed to tackle instabilities in extended systems, we will describe the inhomogeneous equilibrium patterns that we observe.

\section{SUBCRITICAL ZIGZAG TRANSITION IN THE THERMODYNAMIC LIMIT}

Assuming the thermodynamic limit justifies a description of the system as a continuous medium described by partial differential equations. According to our simulations results, the equilibrium patterns may be inhomogeneous, with a zigzag height that depends on the particle position. Therefore, a quantitative description of the inhomogeneous patterns, including nonlinear corrections, may be feasible with slowly varying space and time functions. This is done in this section, using theoretical tools developed for instabilities in extended systems [32].

\section{A. Translational invariance and coupling with the phase mode}

A zigzag pattern is invariant under translations $x \longrightarrow(x+$ $\phi)$, where $\phi$ is a real constant. Thus, in the long-wavelength and low-frequency limit, the energy required by a time- and spacedependent field $\phi(x, t)$ vanishes. Indeed, the field $\phi(x, t)$ is the Goldstone mode linked to the breaking of the translational invariance by the actual zigzag pattern. Very near the pitchfork bifurcation, there is also a soft mode linked to the bifurcation itself. It is a modulated zigzag pattern $h(x, t)$, slowly varying with space and time. In Ref. [32], it was shown that the normal form at the bifurcation results from the nonlinear coupling between these two soft modes.

In our calculations, we assume the vicinity of the zigzag transition, $|\epsilon| \ll 1$. This suggests that the zigzag height $h$ scales as $\epsilon^{1 / 2}$. We therefore write $h=\epsilon^{1 / 2} H$, where $H$ is of order 1 . Then the slow time and space variables are chosen to recover the acoustic modes in the low-frequency and long-wavelength limit (see Appendix B). We thus take $T=\epsilon^{1 / 2} t$ and $X=\epsilon^{1 / 2} x$. The scale of the space variable defines the scale of the field $\phi=\epsilon^{1 / 2} \Phi$, where $\Phi$ is of order 1. The fields $H$ and $\Phi$ depend on the slow variables $X$ and $T$. As in Ref. [32], we will call $\Phi(X, T)$ the phase field [34].

As shown in Fig. 1, we consider only interactions up to second neighbors. It is the simplest model, since nearestneighbor interactions only are insufficient to stabilize the zigzag pattern [16], while taking into account the second neighbors prohibits the zigzag collapse. With this assumption and our previous scaling choices, the relevant distances read

$$
\begin{gathered}
A_{0} B_{0}=\sqrt{\left[d+\epsilon^{1 / 2} \Phi\left(X+\epsilon^{1 / 2} d\right)-\epsilon^{1 / 2} \Phi(X)\right]^{2}+\epsilon\left[H\left(X+\epsilon^{1 / 2} d\right)+H(X)\right]^{2}}, \\
A_{0} A_{1}=\sqrt{\left[2 d+\epsilon^{1 / 2} \Phi\left(X+2 \epsilon^{1 / 2} d\right)-\epsilon^{1 / 2} \Phi(X)\right]^{2}+\epsilon\left[H\left(X+2 \epsilon^{1 / 2} d\right)-H(X)\right]^{2}} .
\end{gathered}
$$

Expanding the interaction energy $U\left(A_{0} B_{0}\right)+U\left(A_{0} A_{1}\right)$ up to $\epsilon^{2}$, and setting $\partial \Phi / \partial X \equiv \Phi_{X}$, we get

$$
\begin{aligned}
\widetilde{E} \approx & U(d)+U(2 d)+\epsilon H^{2}\left(\frac{\beta}{2}+\frac{2 U^{\prime}(d)}{d}\right)+\epsilon^{2}\left\{\left[U^{\prime \prime}(d)-\frac{U^{\prime}(d)}{d}\right]\left(\frac{2 H^{4}}{d^{2}}+2 H^{2} \Phi_{X}\right)\right. \\
& \left.+H H_{X X} d U^{\prime}(d)+\Phi_{X}^{2} \frac{d^{2}}{2}\left[U^{\prime \prime}(d)+4 U^{\prime \prime}(2 d)\right]+H_{X}^{2} \frac{d}{2}\left[U^{\prime}(d)+2 U^{\prime}(2 d)\right]\right\} .
\end{aligned}
$$

The interaction energy $\widetilde{E}$ gives the Lagrangian density $\mathcal{L}=m \epsilon^{2}\left(\Phi_{T}^{2}+H_{T}^{2}\right) / 2-\widetilde{E}$. Notice that we have omitted a term proportional to $\Phi_{X}$ at order $\epsilon$, and the two terms

$$
\epsilon^{3 / 2}\left[2 H H_{X} U^{\prime}(d)+\frac{d^{2}}{2}\left(U^{\prime}(d)+4 U^{\prime}(2 d)\right) \Phi_{X X}\right]=\epsilon^{3 / 2} \frac{\partial}{\partial X}\left[H^{2} U^{\prime}(d)+\frac{d^{2}}{2}\left(U^{\prime}(d)+4 U^{\prime}(2 d)\right) \Phi_{X}\right]
$$


and

$$
\epsilon^{2} \Phi_{X X X} \frac{d^{3}}{6}\left(U^{\prime}(d)+8 U^{\prime}(2 d)\right)=\epsilon^{2} \frac{\partial}{\partial X}\left[\Phi_{X X} \frac{d^{3}}{6}\left(U^{\prime}(d)+8 U^{\prime}(2 d)\right)\right],
$$

because they all are exact $X$ derivatives and thus do not contribute to the Lagrange equations [35]. The Lagrange equations for the fields $H(X, T)$ and $\Phi(X, T)$ are thus

$$
\begin{gathered}
m \epsilon^{2} H_{T T}=-\epsilon\left(\beta+\frac{4 U^{\prime}(d)}{d}\right) H-\epsilon^{2}\left[U^{\prime \prime}(d)-\frac{U^{\prime}(d)}{d}\right]\left(\frac{8}{d^{2}} H^{3}+4 H \Phi_{X}\right)-\epsilon^{2} H_{X X} d\left[U^{\prime}(d)-2 U^{\prime}(2 d)\right], \\
m \epsilon^{2} \Phi_{T T}=\epsilon^{2} d^{2}\left[U^{\prime \prime}(d)+4 U^{\prime \prime}(2 d)\right] \Phi_{X X}+4 \epsilon^{2}\left[U^{\prime \prime}(d)-\frac{U^{\prime}(d)}{d}\right] H H_{X} .
\end{gathered}
$$

This system of equations exhibits the coupling between the soft zigzag mode $H(X, T)$ due to the vicinity of the pitchfork bifurcation $[14,16]$ and the phase mode $\Phi(X, T)$ due to translational invariance, according to the mechanism described in Refs. [32,33]. It leads to the correct acoustic spectrum in the long-wavelength limit (see Appendix B), which justifies $a$ posteriori the scaling choices for the slow scales $X$ and $T$.

Note also that the terms in Eqs. (7) and (8) satisfy all symmetry requirements $[27,32,33,36,37]$. The translational invariance $x \longrightarrow x+\Phi_{0}$ is preserved since only the $X$ derivatives of $\Phi$ appear. The fact that all terms in (7) are odd in $H$ while all terms in (8) are even in $H$ reflects the invariance of the zigzag pattern under the transform $(H, \Phi) \longrightarrow(-H, \Phi)$. It is easily checked that the equations are invariant under the $X$ parity symmetry $(H, X, \Phi) \longrightarrow(H,-X,-\Phi)$.

As a consistency check, we may also verify that these equations include the dependency of the transition threshold on the interparticle distance. We remark that Eq. (7) admits the special solution $\Phi_{X}=\Phi_{X}^{0}=$ Cste and $H_{X}=0$, which corresponds to a renormalization of the mean interparticle distance, which becomes $d_{\text {eff }}=d+\epsilon \Phi_{X}^{0} d$. By considering the terms of (7) that are proportional to $H$, we see that their physical significance is indeed that the instability threshold has changed, to become

$$
\begin{aligned}
\beta_{Z Z}\left(d_{\mathrm{eff}}\right) & =-\frac{4 U^{\prime}\left(d+\epsilon \Phi_{X}^{0} d\right)}{d+\epsilon \Phi_{X}^{0} d} \\
& =-\frac{4 U^{\prime}(d)}{d}-4 \epsilon \Phi_{X}^{0}\left[U^{\prime \prime}(d)-\frac{U^{\prime}(d)}{d}\right]+O\left(\epsilon^{2}\right) .
\end{aligned}
$$

\section{B. Subcritical normal form of the bifurcation}

Most importantly, Eqs. (7) and (8) provide the nonlinear mechanism responsible for the change in the character of the bifurcation. Searching for a stationary solution $H_{T}=0, \Phi_{T}=$ 0 , Eq. (8) is readily integrated to give

$$
\Phi_{X}=-\frac{2\left[d U^{\prime \prime}(d)-U^{\prime}(d)\right]}{d^{3}\left[U^{\prime \prime}(d)+4 U^{\prime \prime}(2 d)\right]} H^{2} \equiv-\alpha \frac{H^{2}}{d^{2}} \quad \text { with } \quad \alpha>0 .
$$

Injecting this expression in (7), and recalling $\beta+4 U^{\prime}(d) / d=$ $\left[4 U^{\prime}(d) / d\right] \epsilon$, we get that the height of a zigzag pattern

$$
\begin{aligned}
& H=C \text { ste is given by } \\
& \underbrace{U^{\prime}(d)}_{<0} \frac{H}{d}=-4 \underbrace{\left[d U^{\prime \prime}(d)-U^{\prime}(d)\right]}_{>0}(2-\alpha) \frac{H^{3}}{d^{3}} \equiv \frac{1}{4} g_{3} \frac{H^{3}}{d^{3}} .
\end{aligned}
$$

Without the coupling with the phase field $\Phi, \alpha=0$, and we recover the supercritical pitchfork bifurcation that can be deduced from (1). When the coupling is taken into account, the coefficient $(2-\alpha)$ may change sign, so that the pitchfork bifurcation becomes subcritical. This happens when the interaction potential is of short range (see Appendix C). For the modified Bessel interaction used in our simulations, we have $(2-\alpha)<0$ when $d / \lambda_{0}>1.58$. Our simulations (see Sec. II) are done with $d / \lambda_{0} \approx 3.92$. It is thus possible to observe a change in the character of the bifurcation because of the coupling with the phase field, and in Sec. IV we will show that this is indeed the case. In Appendix C, we also show that for the Coulomb potential $g_{3}<0$, which is consistent with the fact that the zigzag pattern is stable in a Coulomb system [16,17].

When $g_{3}>0$, the bifurcation is subcritical and it becomes necessary to calculate higher-order terms in Eq. (7) in order to get stable solutions above the bifurcation threshold and thus saturate the instability. We thus carry on the expansion of the energy (4) to higher-order terms. We find a $\epsilon^{5 / 2}$ term that is a total $X$ derivative, and it may thus be dropped [38]. Then the $\epsilon^{3}$ term is rather complicated. We keep only the terms in $H^{5}$, $H^{3} \Phi_{X}$, and $H \Phi_{X}^{2}$. When gathered with the help of Eq. (9), they give a single term in $H^{5}$. Higher derivatives of $H$ happen, but for the sake of simplicity we neglect them. We will see in the next section that this crude approximation is numerically excellent.

We now turn back to the original variable $h$, and we use the interparticle distance $d$ as the unit length. The normal form of the bifurcation is thus

$$
m h_{T T}=\beta_{Z Z} \epsilon h+g_{3} h^{3}-g_{5} h^{5}+g_{1} h_{X X},
$$

where the coefficient $g_{3}$ is defined in (10), and where

$$
\begin{aligned}
\beta_{Z Z} \equiv & -4 U^{\prime}(d)>0, \\
g_{1} \equiv & -\left[U^{\prime}(d)-2 U^{\prime}(2 d)\right]>0, \\
g_{5} \equiv & 2\left\{2\left(6-6 \alpha+\alpha^{2}\right)\left[U^{\prime}(d)-d U^{\prime \prime}(d)\right]\right. \\
& \left.+(2-\alpha)^{2} d^{2} U^{\prime \prime \prime}(d)\right\}>0 .
\end{aligned}
$$



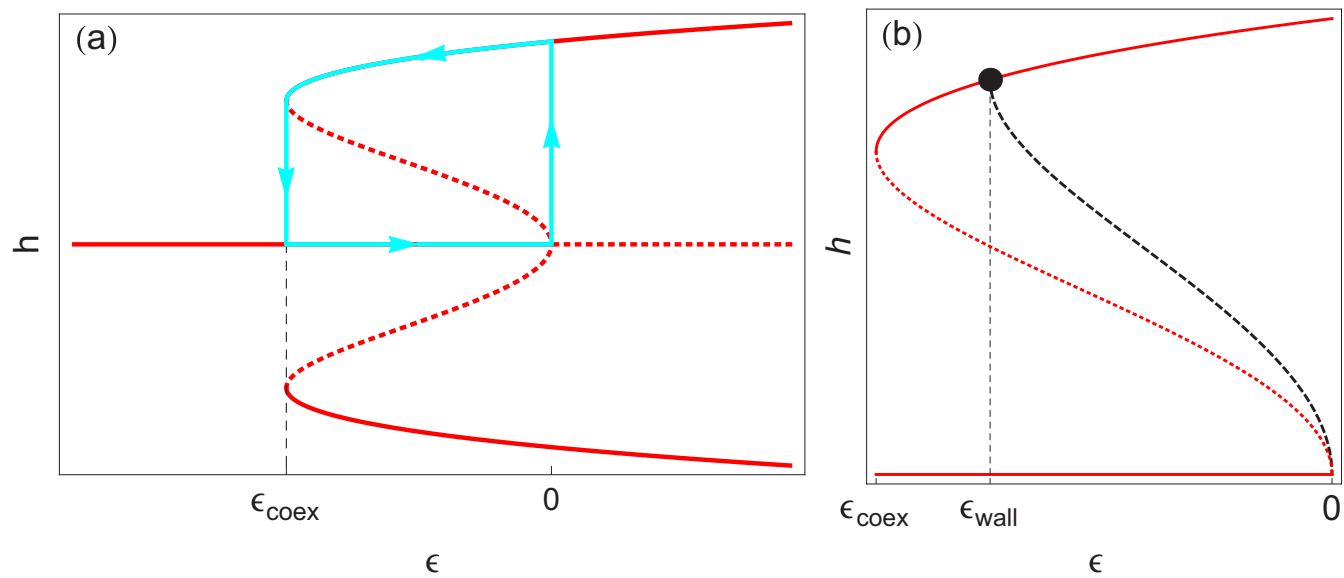

FIG. 6. (Color online) (a) In red (dark gray), we show the bifurcation diagram corresponding to (13). Solid (dotted) lines represent stable (unstable) solutions. In cyan (light gray), we sketch an hysteresis cycle. (b) : Homogeneous zigzag height $h$ (for $h>0$ only) as a function of the distance to threshold $\epsilon$. Red solid line, stable solutions $h=0$ and $h=h_{+}$. Red dotted line, unstable solution $h=h_{-}$[see (13)]. Dashed black line, maximum height of bubbles solutions $h=h_{\text {buble }}$ [see (18)]. The intersection of the lines $h_{\text {bubble }}$ and $h_{+}$corresponds to a wall solution and is emphasized by the large black dot.

The signs of the coefficients are valid for the conditions of our simulations (their respective numerical values are $\beta_{Z Z}=$ $115.8, g_{1}=28.2, g_{3}=208$, and $\left.g_{5}=1489\right)$. The coefficient $g_{3}$ is positive, so that the normal form (11) describes a subcritical pitchfork bifurcation. The sign of the coefficient $g_{5}$ ensures the stability of the bifurcated solution.

The homogeneous patterns are such that $h_{X}=0$. With this assumption, the equilibria of (11) are readily found to be

$$
\begin{aligned}
h & =0, \quad \text { stable when } \epsilon<0, \\
h_{ \pm}^{2} & =\frac{1}{2 g_{5}}\left(g_{3} \pm \sqrt{g_{3}^{2}+4 \epsilon \beta_{Z Z} g_{5}}\right) .
\end{aligned}
$$

The solutions $\pm h_{-}$exist if $\epsilon_{\mathrm{coex}} \equiv-g_{3}^{2} /\left(4 \beta_{Z Z} g_{5}\right) \leqslant \epsilon \leqslant 0$, while the solutions $\pm h_{+}$are defined for $\epsilon_{\text {coex }} \leqslant \epsilon$. The linear stability analysis is straightforward, and it shows that the solutions $\pm h_{-}$are unstable whereas the solutions $\pm h_{+}$are stable. The bifurcation diagram for the normal form (11) is that of a subcritical pitchfork bifurcation, as shown in Fig. 6. It allows for the coexistence between bifurcated and nonbifurcated solutions and the hysteresis cycles for $\epsilon_{\text {coex }} \leqslant$ $\epsilon \leqslant 0$, in agreement with the simulation results of Sec. II.

\section{Stationary inhomogeneous patterns}

Here we search for stationary inhomogeneous patterns. The stationary solutions of Eq. (11) are such that $h_{T}=0$ and are thus solutions of

$$
g_{1} h_{X X}=-\frac{d W}{d h}=-\frac{d}{d h}\left[\frac{1}{2} \epsilon \beta_{Z Z} h^{2}+\frac{1}{4} g_{3} h^{4}-\frac{1}{6} g_{5} h^{6}\right] \text {. }
$$

This equation is similar to the conservative equation of motion of a fictitious particle of mass $g_{1}$ in the potential $W(h)$, with the variable $X$ playing the role of time [39].

For $\epsilon_{\text {coex }} \leqslant \epsilon \leqslant 0$, several inhomogeneous solutions $h_{X} \neq$ 0 exist. They may be described with the help of the plots of $W(h)$ as a function of $h$, shown in Figs. 7, 8, and 9. For all values of $\epsilon$, we have $W(0)=0$, and under the assumption $\epsilon_{\text {coex }} \leqslant \epsilon \leqslant 0$ this is a local maximum of $W(h)$ because the aligned particles $(h=0)$ are a stable homogeneous solution of (11). The unstable homogeneous solution $h_{-}$corresponds, in the mechanical analogy, to a local minimum of $W(h)$, and the stable homogeneous solution $h_{+}$corresponds to a local maximum. An inhomogeneous pattern is defined, in the mechanical analogy, by its conserved "energy" $W_{0}$.

\section{Walls}

The simplest inhomogeneous pattern to calculate is a wall, which links an homogeneous solution $h=0$ to a bifurcated solution $h=h_{+}$, where $h_{+}$is given by (13). A necessary condition for this coexistence is thus $W\left(h_{+}\right)=W(0)=0$, which is only possible for a particular value $\epsilon_{\text {wall }}$ [see Fig. 6(b)], which is easily found to be

$$
\epsilon_{\mathrm{wall}}=-\frac{3}{16} \frac{g_{3}^{2}}{\beta_{Z Z} g_{5}}, \quad h_{\mathrm{wall}} \equiv h_{+}\left(\epsilon_{\mathrm{wall}}\right)=\sqrt{\frac{3 g_{3}}{4 g_{5}}} .
$$

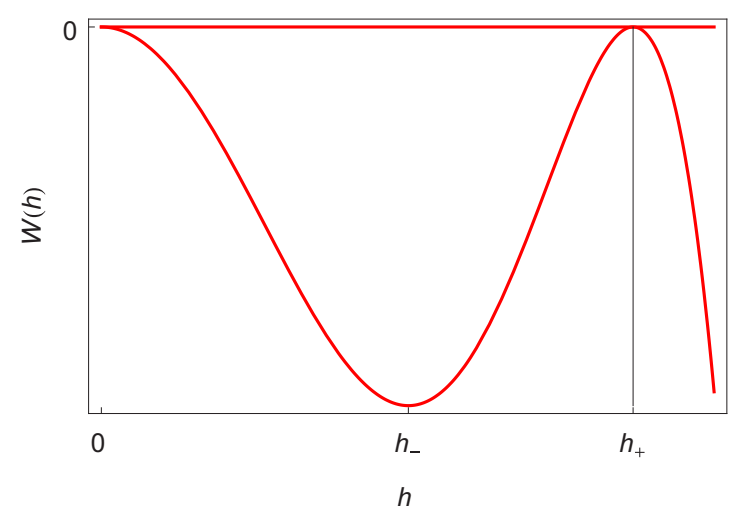

FIG. 7. (Color online) Plot of the dimensionless potential $W(h)$, Eq. (14), as a function of the dimensionless zigzag height $h$. We indicate the location $h_{-}$of the local minimum and the location $h_{+}$of the local maximum. The plot is done for the particular value $\epsilon=\epsilon_{\text {wall }}$, such that $W\left(h_{+}\right)=W(0)=0$. 


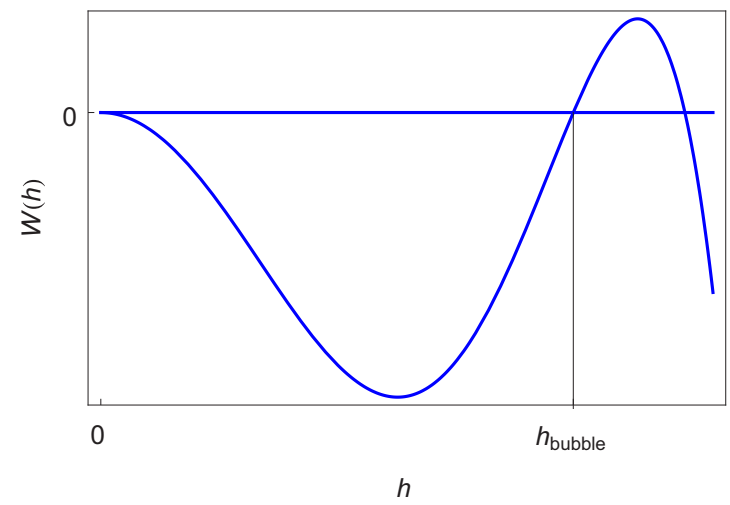

FIG. 8. (Color online) Plot of the dimensionless potential $W(h)$ for $\epsilon_{\text {wall }}<\epsilon<0$ [see Eq. (14)], as a function of the dimensionless zigzag height $h$. The line $W(h)=0$ defines a bubble of height $h_{\text {bubble }}$.

Translating the mechanical analogy in the context of patterns, we see that $h \rightarrow 0$ for $X \rightarrow-\infty$ and $h \rightarrow h_{\text {wall }}$ for $X \rightarrow+\infty$, which justify calling this pattern a wall. Knowing two double roots of $W(h)$, we rewrite it in a particularly simple form,

$$
W_{\text {wall }}(h)=-\frac{g_{5}}{6} h^{2}\left(h_{\text {wall }}^{2}-h^{2}\right)^{2} .
$$

The function $W_{\text {wall }}(h)$ is plotted in Fig. 7. Equation (14) is then readily integrated to give the wall solution as

$$
\frac{h(x)}{d}=h_{\mathrm{wall}} \frac{\exp (\kappa x / d)}{\sqrt{1+\exp (2 \kappa x / d)}}, \quad \text { where } \quad \kappa \equiv \frac{\sqrt{3}}{4} \frac{g_{3}}{\sqrt{g_{1} g_{5}}},
$$

where we have restored the physical units, and we choose for the wall the position $x=0$. A single wall cannot be observed in periodic systems because it obviously violates the periodicity condition.

\section{Bubbles}

When $\epsilon_{\text {wall }}<\epsilon<0$, the maximum of $W(h)$ is strictly positive (see Fig. 8). Let us consider the "trajectory" that corresponds to the constant "energy" $W(0)=0$. In this case,

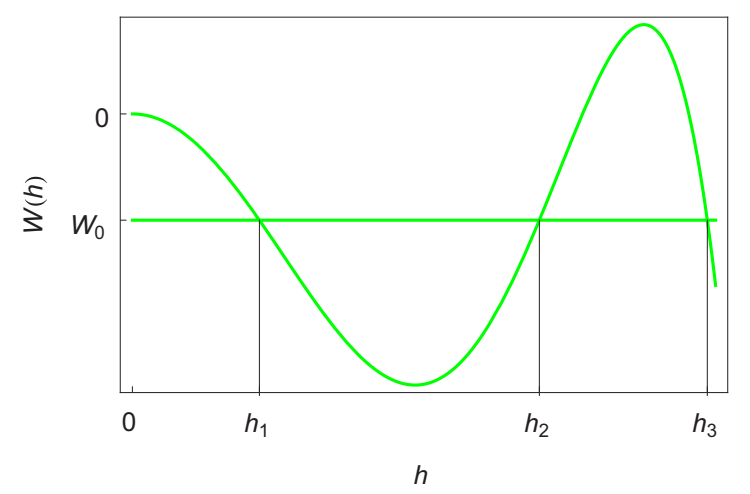

FIG. 9. (Color online) Plot of the dimensionless potential $W(h)$ for $\epsilon_{\text {wall }}<\epsilon \leqslant 0$, Eq. (14), as a function of the dimensionless zigzag height $h$. The straight line of ordinate $W_{0}$ defines a nonlinear modulation between the minimum height $h_{1}$ and the maximum height $h_{2}$. the fictitious particle takes an infinite "time" to get away from the unstable equilibrium position $h=0$, then a finite "time" to reach a maximum height $h_{\text {bubble }}$ such that $W\left(h_{\text {bubble }}\right)=0$, then an infinite "time" to come back to its initial position $h=0$. In the framework of the zigzag transition, it corresponds to a localized zigzag of height $h_{\text {bubble }}$ surrounded by aligned particles with $h(X \rightarrow \pm \infty) \rightarrow 0$. We call this pattern a bubble.

The bubble height $h_{\text {bubble }}$ is the solution of $W\left(h_{\text {bubble }}\right)=0$. The real nonzero roots of this equation are

$$
h_{b \pm}^{2}=\frac{3}{2 g_{5}}\left(\frac{g_{3}}{2} \pm \sqrt{\frac{g_{3}^{2}}{4}+\frac{4}{3} \epsilon g_{5} \beta_{Z Z}}\right) .
$$

We have $h_{b-} \leqslant h_{b+}$, the equality corresponding to the case of a wall. The bubble height is thus $h_{\text {bubble }}=h_{b-}$, as shown in Fig. 8. In Fig. 6(b), we display the evolution of the bubble height with $\epsilon$.

The potential $W(h)$ may be written

$$
W_{\text {bubble }}(h)=\frac{g_{5}}{6} h^{2}\left(h^{2}-h_{b-}^{2}\right)\left(h_{b+}^{2}-h^{2}\right) .
$$

A bit of algebra is sufficient to integrate (14) with this potential, giving

$$
\frac{h(x)}{d}=\frac{h_{b-} h_{b+}}{\sqrt{\left(h_{b+}^{2}-h_{b-}^{2}\right) \cosh ^{2}\left(\kappa^{\prime} x / d\right)+h_{b-}^{2}}},
$$

where

$$
\kappa^{\prime} \equiv h_{b-} h_{b+} \sqrt{\frac{g_{5}}{3 g_{1}}}=\sqrt{\frac{-\epsilon \beta_{Z Z}}{g_{1}}} .
$$

When $\epsilon \rightarrow \epsilon_{\text {wall }}$, it is easy to see that $\kappa^{\prime} \rightarrow \kappa$, which means that the spatial variation of height for a bubble becomes equal to that for a wall. In this limit, the bubble height becomes independent of $\epsilon$, but the bubbles are more and more flat [40]. An example is shown in the second plot of Fig. 10.

\section{Modulated zigzag patterns}

The minimum of $W(h)$ corresponds to the unstable homogeneous solution $\pm h_{-}$[see Eq. (13)]. Let $W_{0}$ be a negative constant such that $0>W_{0}>W\left( \pm h_{-}\right)$. The equation $W(h)=$ $W_{0}$ thus admits three real and positive solutions $h_{1}<h_{2}<h_{3}$, defined graphically in Fig. 9. In the mechanical analogy, it corresponds to a nonlinear periodic motion around $\pm h_{-}$, and it describes a nonlinear modulation of the zigzag pattern. Its minimum height is given by $h_{1}$ and its maximum height is given by $h_{2}$.

In Appendix D, we show that the modulated patterns may be expressed in terms of Jacobi elliptic functions, as

$$
\frac{h(x)}{d}=\frac{h_{1} h_{2}}{\sqrt{h_{2}^{2} \operatorname{cn}^{2}\left(\frac{x-\Lambda K(Q)}{d \Lambda}, Q\right)+h_{1}^{2} \operatorname{sn}^{2}\left(\frac{x-\Lambda K(Q)}{d \Lambda}, Q\right)}},
$$



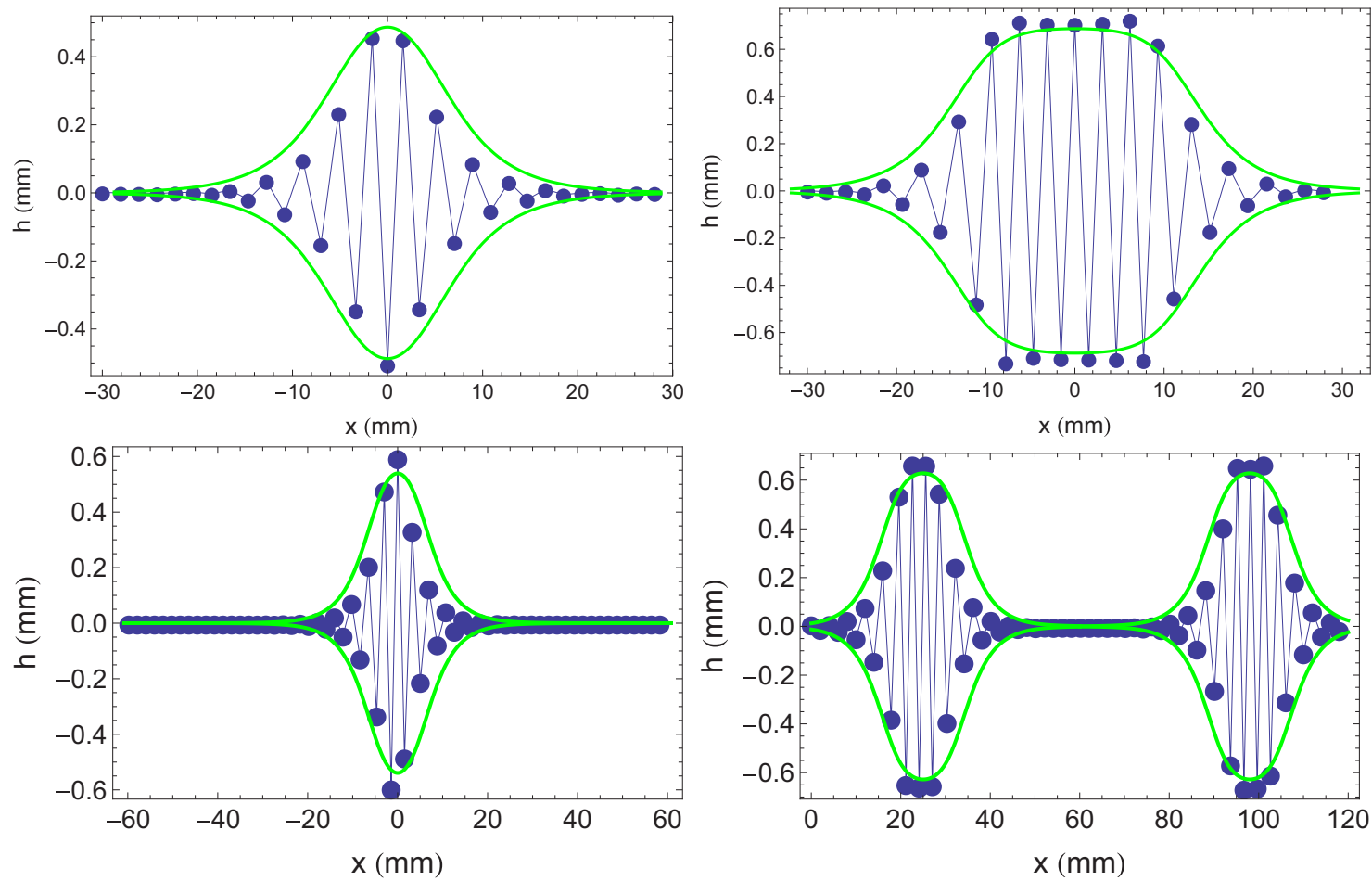

FIG. 10. (Color online) Comparison between simulations (blue points) and theoretical predictions (thick solid lines) for localized zigzag equilibrium patterns (bubbles). From top to bottom and left to right, $N=32$ and $\epsilon_{\text {num }}=0.07, N=32$ and $\epsilon_{\text {num }}=0.48$ [see the lower inset in Fig. 3 (a)], $N=64$ and $\epsilon_{\text {num }}=0.0264$, and $N=64$ and $\epsilon_{\text {num }}=0.261$. For clarity, the patterns maxima are placed at the center of the frame even if they can be anywhere in the periodic cell.

where $K(\cdot)$ is the complete elliptic integral of the first kind, and where

$$
\Lambda \equiv\left[\left(h_{3}^{2}-h_{1}^{2}\right) h_{2}^{2} \frac{g_{5}}{3 g_{1}}\right]^{-1 / 2}, \quad Q \equiv \frac{h_{3} \sqrt{h_{2}^{2}-h_{1}^{2}}}{h_{2} \sqrt{h_{3}^{2}-h_{1}^{2}}} .
$$

The solution (22) is such that the maximum height takes place at $x=0$, and $2 \Lambda K(Q)$ is the wavelength of the spatial modulation. As explained in Appendix D, the modulated pattern is fully determined by the two parameters $\epsilon$ and $h_{2}$, which is particularly well suited to our analysis.

\section{FINITE-SIZE EFFECTS}

In the preceding section, we found a mechanism that explains why the zigzag transition actually is a subcritical bifurcation in translationally invariant infinite systems with short-range interactions. The key phenomenon is the nonlinear coupling between the soft mode at the zigzag transition and the phase mode linked to the translational invariance. This phenomenon also happens in finite systems with periodic boundary conditions because of their rotational invariance.

The subcritical bifurcation diagram of Fig. 6 is also relevant for periodic systems with short-range interactions. It is consistent with the observations reported on in Sec. II. The agreement is also quantitative. For instance, the hysteresis cycles displayed in Fig. 5 are consistent with a limiting value of the stiffness gap $\beta_{\text {up }}-\beta_{\text {down }}$ equal to $\epsilon_{\text {coex }}(d) \beta_{Z Z}(d)$ when $N \rightarrow \infty$.
In the simulations, the inhomogeneous patterns are observed when the stiffness $\beta_{\text {num }}$ of the confining potential is such that $\beta_{\text {num }}<\beta_{Z Z}(d)$. This defines a positive parameter $\epsilon_{\text {num }}$ as $\beta_{\text {num }}=\beta_{Z Z}(d)\left(1-\epsilon_{\text {num }}\right)$. In contrast, the inhomogeneous patterns are predicted for negative values of $\epsilon$. In this section, we will explain how to get rid of this apparent discrepancy by taking into account finite-size effects.

\section{A. Localized zigzag patterns}

Let us first focus on the bubbles. In these patterns, we can measure the distance between any two aligned particles. This distance $d_{\text {eff }}$ is found to be constant, and such that $d_{\text {eff }}>d$ because the particles in the localized zigzag allow the others to expand. From this interparticle distance, we define an effective bifurcation parameter $\epsilon_{\text {eff }}$,

$$
\epsilon_{\mathrm{eff}}=1-\frac{\beta_{\text {num }}}{\beta_{Z Z}\left(d_{\mathrm{eff}}\right)} .
$$

We will interpret a bubble observed in the finite periodic system as a bubble in an infinite system, with an interparticle distance $d_{\mathrm{eff}}$, when the distance to threshold is $\epsilon_{\mathrm{eff}}$.

Notice that for consistency $\epsilon_{\text {eff }}$ must be negative, with $\epsilon_{\text {wall }}\left(d_{\text {eff }}\right)<\epsilon_{\text {eff }} \leqslant 0$, because it is a necessary condition for the existence of bubbles in infinite systems. This is indeed what happens, as shown by the data summarized in Table I. The effective bifurcation parameter becomes negative because $d_{\text {eff }}>d$ and the zigzag threshold $\beta_{Z Z}(d)=-4 U^{\prime}(d) / d$ is a strongly decreasing function of $d$. So, once the value of $d_{\mathrm{eff}}$ is measured on the simulation data, the bubble is completely 
TABLE I. Summary of the effective distance to the threshold, $\epsilon_{\text {eff }}$, and of the relative discrepancy $|L-\widetilde{L}| / L$ corresponding to several localized excitations. The theoretical expectation $\widetilde{L}$ is given by Eq. (25) in all cases except the last one, which concerns two identical bubbles (see Fig. 10), thus requiring the change $\alpha \rightarrow 2 \alpha$. For the sake of comparison, $d=1.875 \mathrm{~mm}$.

\begin{tabular}{cccccc}
\hline \hline & $\begin{array}{c}d_{\text {eff }} \\
N\end{array}$ & & & $|L-\widetilde{L}| / L$ & $\left(N d_{\text {eff }}-L\right) / L$ \\
$(\%)$ & $\epsilon_{\text {num }}$ & $\epsilon_{\text {eff }}$ & $(\%)$ & 4.9 \\
\hline 16 & 1.967 & 0.206 & -0.037 & 0.17 & 1.9 \\
32 & 1.910 & 0.070 & -0.0230 & 0.02 & 2.9 \\
32 & 1.929 & 0.110 & -0.042 & 0.09 & 13.1 \\
32 & 2.120 & 0.480 & -0.052 & 0.04 & 1.2 \\
64 & 1.897 & 0.0284 & -0.036 & 0.01 & 1.0 \\
64 & 1.893 & 0.021 & -0.032 & 0.05 & 6.5 \\
64 & 1.996 & 0.261 & -0.050 & 0.30 & \\
\hline \hline
\end{tabular}

determined with the help of Eqs. (20) and (18), where all coefficients $g_{i}$, defined in Eqs. (10) and (12), are calculated for an infinite system in which the aligned particles are separated by $d_{\text {eff }}$.

This analysis is done on several simulations data, and the results are shown in Fig. 10. In each case, the effective distance $d_{\text {eff }}$ is easily measured, and the comparison between the simulations and the bubbles expected from the theoretical analysis evidences an excellent agreement [41]. In the last plot of Fig. 10, the same value of $d_{\mathrm{eff}}$ is used for both bubbles, which have the same height. Equilibria with several bubbles are sometimes observed, and in all cases all bubbles have the same height, which is consistent with our analysis.

The comparison illustrated in Fig. 10 provides a quantitative test of the mechanism expressed by Eq. (9). The physical significance of the phase field $\Phi$ is a shift in the interparticle distance, which becomes $d_{\mathrm{eff}}\left(1+\Phi_{X}\right)$. Equation (9) relates the phase gradient $\Phi_{X}$ to the local zigzag amplitude. Physically, it expresses that the dilatation of the aligned particles is compensated by the height $h(X)$ of the bubble. This provides a second quantitative test of the coupling between the soft mode at the bifurcation and the Goldstone mode linked to rotational symmetry breaking that is at the basis of the calculations of Sec. III. Indeed, the total length $\widetilde{L}$ occupied by a bubble is

$$
\begin{aligned}
\widetilde{L} & =d_{\text {eff }}\left(N-\alpha \int_{-\infty}^{\infty} \frac{h(X)^{2}}{d_{\text {eff }}^{2}} d X\right) \\
& =d_{\text {eff }}\left[N-\frac{2 \alpha h_{b-} h_{b+}}{\kappa^{\prime}} \operatorname{arctanh}\left(\frac{h_{b-}}{h_{b+}}\right)\right] .
\end{aligned}
$$

For consistency, this length $\widetilde{L}$ has to be identical to the period $L$ of our simulated cell. In Table I, we calculate $|L-\widetilde{L}| / L$ for several bubbles, and we see that it is indeed very small. Since $d_{\text {eff }}$ is rather close to $d$, we compare it to $\left(N d_{\text {eff }}-L\right) / L$. We see that the calculated longitudinal width $\widetilde{L}$ is a much better estimate of the bubble width than the rough approximation $N d_{\text {eff }}$. The last line of Table I corresponds to the two-bubble excitation of Fig. 10, so that we have consistently doubled the contribution of the integral in (25) in this last case.

\section{B. Modulated zigzag patterns}

We also observe modulated patterns, for which the zigzag height varies from a minimum $h_{1}>0$ to a maximum value
TABLE II. Summary of the effective distance to the threshold, $\epsilon_{\text {eff }}$, and of the relative discrepancy $|L-\widetilde{L}| / L$ corresponding to several modulated excitations. The theoretical expectation $\widetilde{L}$ is given

\begin{tabular}{|c|c|c|c|c|c|c|}
\hline$N$ & $\begin{array}{c}d_{\mathrm{eff}} \\
(\mathrm{mm})\end{array}$ & $\epsilon_{\text {num }}$ & $\epsilon_{\text {eff }}$ & $\begin{array}{c}h_{2} \\
(\mathrm{~mm})\end{array}$ & $\begin{array}{c}|L-\widetilde{L}| / L \\
(\%)\end{array}$ & $\begin{array}{c}\left(N d_{\mathrm{eff}}-L\right) / L \\
(\%)\end{array}$ \\
\hline 16 & 1.932 & 0.132 & -0.0257 & 0.306 & 0.35 & 3.1 \\
\hline 16 & 1.943 & 0.150 & -0.0351 & 0.398 & 0.87 & 3.6 \\
\hline 16 & 1.962 & 0.187 & -0.0463 & 0.500 & 2.26 & 4.6 \\
\hline 32 & 1.897 & 0.050 & -0.0137 & 0.239 & 0.12 & 1.2 \\
\hline 64 & 1.881 & 0.017 & -0.0012 & 0.058 & 0.18 & 0.34 \\
\hline 64 & 1.882 & 0.018 & -0.0019 & 0.085 & 0.19 & 0.38 \\
\hline 64 & 1.884 & 0.021 & -0.0047 & 0.137 & 0.18 & 0.47 \\
\hline
\end{tabular}
by Eq. (26). For the sake of comparison, $d=1.875 \mathrm{~mm}$.

$h_{2}$ (see the upper inset in Fig. 3). For modulated patterns, it is impossible to measure directly the parameter $d_{\text {eff }}$, since no particles in the cell are along a straight line. In contrast, the height $h_{2}$ is easily measured from the simulation data. Then the unknown parameter $d_{\mathrm{eff}}$ is determined numerically from a consistency condition, requiring the spatial period of the modulation to be equal to the length $L$ of the periodic box. The effective distance $d_{\text {eff }}$ is thus the real root of the equation $L=2 \Lambda\left(h_{2}, d_{\mathrm{eff}}\right) K\left[Q\left(h_{2}, d_{\mathrm{eff}}\right)\right]$. With the help of (D2) and (24), this equation involves only the unknown parameter $d_{\text {eff. }}$ This is a formally complicated equation that is easily solved numerically. Once $d_{\text {eff }}$ and $h_{2}$ are known, the modulated pattern is completely determined. In Table II, we summarize the calculated values of $d_{\text {eff }}$ and $\epsilon_{\text {eff }}$, defined in Eq. (24). For consistency, the parameter $\epsilon_{\text {eff }}$ must be negative since modulated zigzag patterns happen only when the unstable homogeneous solution $\pm h_{-}$exists. This is indeed the case for all patterns listed in Table II.

The comparison between the observed modulated patterns and the theoretical predictions of Eqs. (22) and (23) is done in Figs. 11 and 12. For the larger systems (32 and 64 particles), the agreement is excellent, and we are able to reproduce the strongly nonsinusoidal pattern in all cases. We display also in Fig. 12 the data for a small system of only 16 particles. Even in this rather unfavorable case, there is a very good agreement between the patterns calculated for an infinite system and the simulation results. It is of interest to emphasize that for the highest value $\epsilon_{\text {num }}=0.206$ in Fig. 12, it is impossible to describe the pattern as a modulation, because there is no real solution to the numerical equation $L=2 \Lambda K(Q)$. It is nevertheless possible to describe it as a bubble, with a slightly different method from the one mentioned in Sec. IV A. We determine the parameter $d_{\mathrm{eff}}$ from the measurement of the pattern height, through Eq. (18).

The same consistency check of Eq. (9) as for bubbles may be done. The total length $\widetilde{L}$ of a modulated pattern, following the same reasoning that led to (25), is

$$
\begin{aligned}
\widetilde{L} & =d_{\text {eff }}\left(N-2 \alpha \int_{0}^{L / 2} \frac{h(X)^{2}}{d_{\text {eff }}^{2}} d X\right) \\
& =d_{\text {eff }}\left[N-2 \alpha h_{1}^{2} \Pi\left(1-\frac{h_{1}^{2}}{h_{2}^{2}}, Q\right)\right],
\end{aligned}
$$



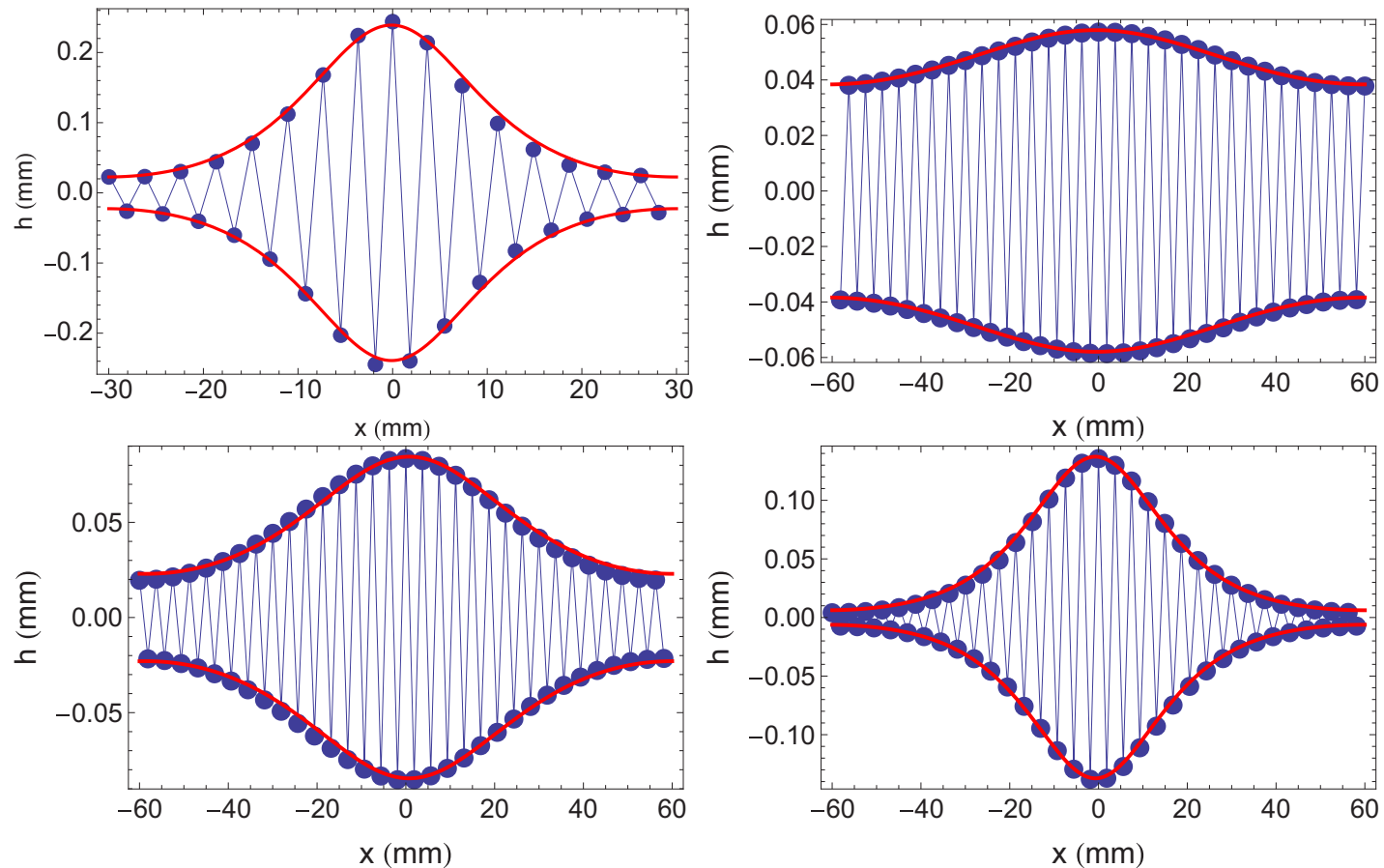

FIG. 11. (Color online) Modulated zigzag patterns. From top to bottom and left to right: $N=32$ and $\epsilon_{\text {num }}=0.05$, and then $N=64$ and $\epsilon_{\text {num }}=0.0173,0.0185$, and 0.0207 . Blue points denote simulation data, while the thick red solid line represents the theoretical prediction, Eq. (22).

where we have used the fact that the spatial period of a modulated pattern is $L$, the symmetry of the pattern, and where we have introduced the complete elliptic integral of the third kind $\Pi(.,$.$) [42]. The results are summa-$ rized in Table II. We see that for these patterns also the error made on the estimated longitudinal width of the pattern is small, and much smaller than the rough estimate $N d_{\text {eff }}$.
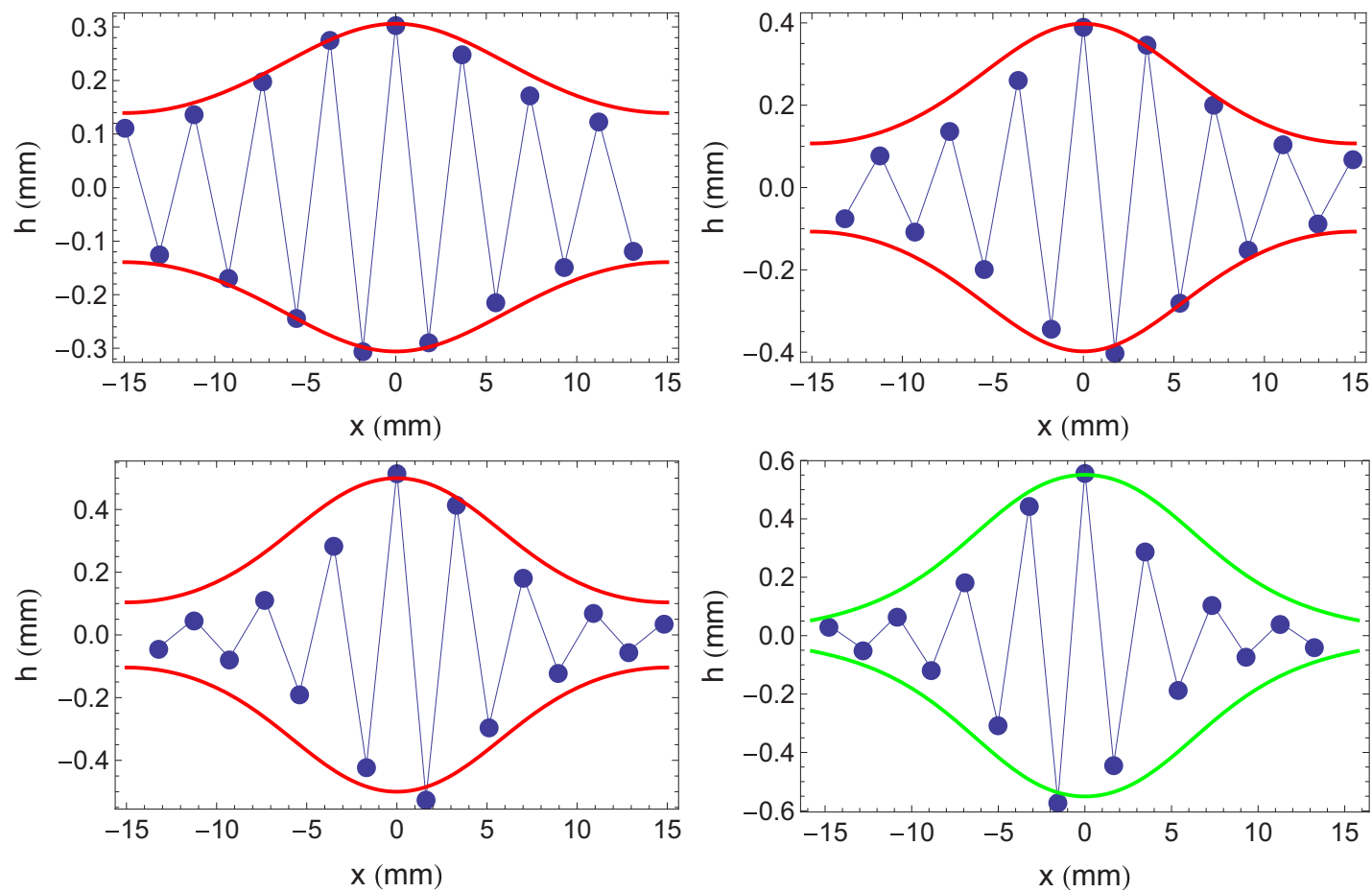

FIG. 12. (Color online) Modulated zigzag patterns for $N=16$. From top to bottom and left to right: $\epsilon_{\text {num }}=0.132,0.150,0.187$, and 0.206 . Blue points denote simulation data, while the thick red solid line represents the theoretical prediction, Eq. (22). The thick green solid line is the theoretical prediction (20), because this pattern cannot be described by a nonlinear modulation. 


\section{CONCLUSION}

In this paper, we discuss the zigzag transition in systems of particles with short-range interactions, which are either infinite or with periodic boundary conditions in the longitudinal axis. With numerical simulations, we give evidence that the equilibrium patterns are not necessarily homogeneous. In sufficiently large systems, the zigzag transition is a first-order configurational transition. The relevant physical mechanism has been identified for instabilities in extended systems, and it results from the nonlinear coupling between the soft mode due to the zigzag transition and a Goldstone mode due to the breaking of the translational or rotational invariance of the system by the zigzag pattern.

In the framework of instabilities in extended systems, the subcritical normal form has been obtained phenomenologically by taking into account the symmetries of the system. In the case of the zigzag transition, the underlying dynamics is explicitly known. It allows us to obtain the normal form in a constructive way, as a systematic asymptotic expansion in the thermodynamic limit, near the bifurcation threshold. We provide analytical expressions of the normal form coefficients and establish the subcritical character of the pitchfork bifurcation.

At the end of the calculation, there is no free fitting parameter, and we are able to perform a quantitative comparison between our simulation data and the theoretical analysis. We describe with excellent precision the inhomogeneous zigzag patterns exhibited in the simulations. The physical significance of the coupling between the soft mode and the phase mode is a shift of the longitudinal interparticle distance, which depends on the local zigzag height. A direct and quantitative test of this mechanism is done and evidences a very good agreement.

\section{ACKNOWLEDGMENTS}

We thank S. Fauve for useful discussions, and for pointing out the relevance of the phase instability mechanism.

\section{APPENDIX A: NUMERICS}

The details on the numerical algorithm can be found in Ref. [43]. For the sake of completeness, we give a short summary below.

We simulate identical point particles located in the plane $(x O y)$, submitted to a thermal bath at temperature $T_{b}$. The thermal bath is accounted for by a damping constant $\gamma$, and by random forces applied on each particle, with the statistical properties of uncorrelated white Gaussian noise. As for most of the experimental configurations for which the zigzag transition is relevant $[3,5,7-11,13,18,44]$, the particles interact with a repulsive potential. We use in all simulations a screened electrostatic interaction $U(r)=U_{0} K_{0}\left(r / \lambda_{0}\right)$ with energy scale $U_{0}$, with a characteristic length $\lambda_{0}$ and where $K_{0}$ is the modified Bessel function of index 0 . To observe equilibrium patterns, the temperature of the thermal bath is such that $U(d) /\left(k_{B} T_{b}\right)<0.01$ in all simulations.

The particles are transversally confined in a quasi-1D geometry by a harmonic potential of stiffness $\beta$, whereas periodic boundary conditions are applied in the longitudinal direction. The dynamics of the system is then simulated by the numerical integration of the coupled Langevin equations. Let $L$ be the length of the simulation cell, and let $N$ be the number of particles. In all simulations, the mean distance $d \equiv L / N$ is kept constant, with a length of $L=60 \mathrm{~mm}$ for $N=32$. The influence of the potential range $\lambda_{0}$ has been discussed in [16]. We set $\lambda_{0} \approx d / 4$ in order to have a linearly unstable zigzag pattern. In some simulations, we vary the stiffness $\beta$ by steps. The typical duration of a stiffness step is $10^{4}$ time steps, and we make typically $10^{3}$ stiffness steps (sometimes up to $10^{5}$ steps) with a relative stiffness variation of $10^{-3}$.

The simulations are done at finite (nonzero) temperature, hence the observed patterns are stable under the thermal fluctuations. To obtain the equilibrium patterns, we average the system motions on a time scale that is intermediate between the time step and the duration of a simulation run. Since this latter is never less than $10^{6}$ time steps, the typical averaging time for an equilibrium pattern is $10^{3}$ time steps.

\section{APPENDIX B: ACOUSTIC MODES OF THE ZIGZAG PATTERN}

In this appendix, we show that Eqs. (7) and (8) correctly describe the acoustic modes of the chain. We recover only the long-wavelength limit, because it is assumed at the beginning of the calculations of Sec. III A that $X$ is a slow variable.

Taking $H=0$, and linearizing (8), we get the sound velocity $c_{s}^{2}=d^{2}\left[U^{\prime \prime}(d)+4 U^{\prime \prime}(2 d)\right] / m$ of the rectilinear chain when nearest-neighbor and next-nearest-neighbor interactions are taken into account [43]. Taking $\Phi=$ Cste, and linearizing (7) for $\beta=\beta_{Z Z}$, we recover the velocity of transverse oscillations $c_{\perp}^{2}=d\left[2 U^{\prime}(2 d)-U^{\prime}(d)\right] / m$ as in Ref. [14].

In the general case, we linearize around the bifurcated homogeneous state $H_{0}$, which from Eq. (1) is seen to be $H_{0}^{2}=\beta_{Z Z} d^{2} /\left(8\left[U^{\prime \prime}(d)-\frac{U^{\prime}(d)}{d}\right]\right)$. Assuming $H(X, T)=$ $H_{0}+\eta(X, T)$ with $|\eta| \ll 1$ and $\left|\Phi_{X}\right| \ll 1$, we obtain from (7) and (8)

$$
\begin{aligned}
m \eta_{T T}= & -2 \beta_{Z Z} \eta-d\left[U^{\prime}(d)-2 U^{\prime}(2 d)\right] \eta_{X X} \\
& -4\left[U^{\prime \prime}(d)-\frac{U^{\prime}(d)}{d}\right] H_{0} \Phi_{X}, \\
m \Phi_{T T}= & d^{2}\left[U^{\prime \prime}(d)+4 U^{\prime \prime}(2 d)\right] \Phi_{X X} \\
& +4\left[U^{\prime \prime}(d)-\frac{U^{\prime}(d)}{d}\right] H_{0} \eta_{X} .
\end{aligned}
$$

Searching the solutions in the form $(\eta, \Phi)=\left(\eta_{0}, \Phi_{0}\right) e^{i(q X-\omega T)}$, we find the eigenfrequencies as the eigenvalues of the matrix,

$$
\frac{1}{m}\left(\begin{array}{cc}
2 \beta_{Z Z}-d\left[U^{\prime}(d)-2 U^{\prime}(2 d)\right] q^{2} & 4 i q\left[U^{\prime \prime}(d)-\frac{U^{\prime}(d)}{d}\right] H_{0} \\
-4 i q\left[U^{\prime \prime}(d)-\frac{U^{\prime}(d)}{d}\right] H_{0} & d^{2}\left[U^{\prime \prime}(d)+4 U^{\prime \prime}(2 d)\right] q^{2}
\end{array}\right) .
$$




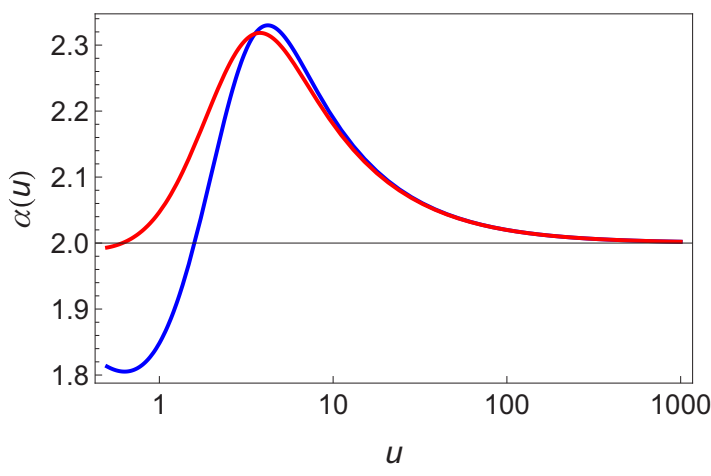

FIG. 13. (Color online) Plot of $\alpha(u)$ (linear scale) as a function of $u$ (log scale) for the modified Bessel function potential (blue curve) and the Yukawa potential (red curve).

This has to be compared with the calculations of Ref. [16], restricted to the second neighbors only, in the long-wavelength limit $\psi=q d \rightarrow 0$, and where all coefficients have to be expanded up to first order in $h_{0}$ only. The acoustic spectrum is then consistently recovered.

The scaling $h_{0}=H_{0} \sqrt{\epsilon}$ is imposed by the pitchfork bifurcation, and we see that the acoustic modes are recovered if $\omega^{2}, q^{2}$, and $h_{0}^{2}$ all are of order $\epsilon$, which is ensured by the scalings of the slow variables $T=\epsilon^{1 / 2} t, X=\epsilon^{1 / 2} x$ as announced in the beginning of Sec. III A.

\section{APPENDIX C: NUMERICAL ESTIMATES OF $\alpha$}

In this appendix, we discuss the numerical value of the parameter $\alpha$ defined in Eq. (9), which is directly linked to the subcritical or supercritical character of the bifurcation in infinite or periodic systems. Let us first consider short-range potentials, such as the modified Bessel function used in the simulations (see Appendix A) and the Yukawa potential. Let $u \equiv d / \lambda_{0}$. For the modified Bessel potential, we get

$$
\alpha(u)=\frac{2 u\left[K_{0}(u)+K_{2}(u)\right]+4 K_{1}(u)}{u\left\{K_{0}(u)+K_{2}(u)+4\left[K_{0}(2 u)+K_{2}(2 u)\right]\right\}} .
$$

Since $K_{n}(u) \sim e^{-u} / \sqrt{2 \pi u}$ for large $u$, we easily see that $\lim _{u \rightarrow \infty} \alpha(u)=2$. The function $\alpha(u)$ is plotted in Fig. 13 . We find that $\alpha \geqslant 2$ for $d / \lambda_{0} \geqslant 1.58$, and that the maximum value of $\alpha$ is 2.33 .

For the Yukawa potential, we get

$$
\alpha(u)=\frac{2\left(u^{2}+3 u+3\right)}{u^{2}+2 u+2+e^{-u}\left(2 u^{2}+2 u+1\right)},
$$

which also gives $\lim _{u \rightarrow \infty} \alpha(u)=2$. The function $\alpha(u)$ is plotted in Fig. 13. We find that $\alpha \geqslant 2$ for $d / \lambda_{0} \geqslant 0.61$, and that the maximum value of $\alpha$ is 2.32 .
The numerical value of $\alpha$ for the modified Bessel potential explains an observation made in Ref. [16]. Here it was shown that if we consider a bubble pattern, the distance between nearest neighbors in and outside the bubble was almost constant. In our description, the particles outside the bubble are at a distance $d_{\text {eff }}$, while those inside the bubble are separated from $\sqrt{\left(d_{\text {eff }}-\alpha h^{2}\right)^{2}+4 h^{2}}$. Since $(\alpha-2)$ is positive but small, and since $h / d$ is also small, the difference between these two distances is less than $1.5 \%$.

It is of interest to consider also the case of the Coulomb potential. It is not difficult to extend our calculations to more than second-neighbor interactions. If we take into account the $n$th cell after the tagged one, one has to expand $A_{0} B_{n}$ and $A_{0} A_{n}$. This amounts to replacing $d$ in Eq. (2) by $(2 n+1) d$, and $2 d$ in Eq. (3) by $2 n d$, and to sum on all interacting particles. The expansion in powers of $\epsilon$ is consistent if $n \epsilon \ll 1$, which excludes an infinite system. Let us therefore consider a finite periodic system of $N_{\max }$ cells. The coefficient $g_{3}$ is now found to be

$$
\begin{aligned}
\frac{g_{3}}{8}= & \frac{\left(\sum_{n=0}^{N_{\max }} \frac{(2 n+1) d U^{\prime \prime}[(2 n+1) d]-U^{\prime}[(2 n+1) d]}{(2 n+1) d}\right)^{2}}{\sum_{n=1}^{N_{\max }} n^{2} d^{2} U^{\prime \prime}(n d)} \\
& -\sum_{n=0}^{N_{\max }} \frac{(2 n+1) d U^{\prime \prime}[(2 n+1) d]-U^{\prime}[(2 n+1) d]}{(2 n+1)^{3} d^{3}} .
\end{aligned}
$$

For the Coulomb potential, $U(r) \propto 1 / r$, the sums in the numerators converge rapidly at large $N_{\max }$ while the sum in the denominator diverges. Hence $g_{3} \leqslant 0$ (with the equality for $N_{\max }=1$ ) and the bifurcation is always supercritical, which is consistent with the linear stability analysis of zigzag patterns [16,17].

\section{APPENDIX D: NONLINEAR MODULATED ZIGZAG}

When $\epsilon_{\text {wall }}<\epsilon<0$, the potential $W(h)$ has a minimum $W_{\text {min }}<0$. Let $W_{0}$ be a constant such that $W_{\text {min }}<W_{0}<0$. Then the equation $W(h)=W_{0}$ has three real roots $h_{1}^{2}<h_{2}^{2}<$ $h_{3}^{2}$, so that we may write

$$
W_{0}-W(h)=\frac{g_{5}}{6}\left(h^{2}-h_{1}^{2}\right)\left(h^{2}-h_{2}^{2}\right)\left(h^{2}-h_{3}^{2}\right) .
$$

This configuration corresponds to a (spatially) periodic nonlinear modulation between the heights $h_{1}$ and $h_{2}$. The algebra is then somewhat tedious, but with the help of Refs. [45,46] we find Eqs. (22) and (23) given in the text.

The roots $h_{i}$ are complicated expressions of $\epsilon$ and $W_{0}$, but two of them are easily expressed as a function of the third, as $h_{1}=h_{m-}$ and $h_{3}=h_{m+}$, where

$$
h_{m \pm}=\frac{1}{2}\left[\frac{3 g_{3}}{2 g_{5}}-h_{2}^{2} \pm \sqrt{\left(h_{2}^{2}-\frac{3 g_{3}}{2 g_{5}}\right)^{2}-4\left(h_{2}^{4}-\frac{3 g_{3}}{2 g_{5}} h_{2}^{2}-\frac{3 \epsilon \beta_{Z Z}}{g_{5}}\right)}\right] .
$$

This is very well suited to our analysis, because $h_{2}$ is the maximum height of the modulated pattern, and thus directly measured. 
[1] I. Waki, S. Kassner, G. Birkl, and H. Walther, Observation of ordered structures of laser-cooled ions in a quadrupole storage ring, Phys. Rev. Lett. 68, 2007 (1992).

[2] D. H. E. Dubin, Theory of structural phase transitions in a trapped Coulomb crystal, Phys. Rev. Lett. 71, 2753 (1993).

[3] J. P. Schiffer, Phase transitions in anisotropically confined ionic crystals, Phys. Rev. Lett. 70, 818 (1993).

[4] D. G. Enzer, M. M. Schauer, J. J. Gomez, M. S. Gulley, M. H. Holzscheiter, P. G. Kwiat, S. K. Lamoreaux, C. G. Peterson, V. D. Sandberg, D. Tupa, A. G. White, R. J. Hughes, and D. V. F. James, Observation of power-law scaling for phase transitions in linear trapped ion crystals, Phys. Rev. Lett. 85, 2466 (2000).

[5] S. Seidelin, J. Chiaverini, R. Reichle, J. J. Bollinger, D. Leibfried, J. Britton, J. H. Wesenberg, R. B. Blakestad, R. J. Epstein, D. B. Hume, W. M. Itano, J. D. Jost, C. Langer, R. Ozeri, N. Shiga, and D. J. Wineland, Microfabricated surface-electrode ion trap for scalable quantum information processing, Phys. Rev. Lett. 96, 253003 (2006).

[6] J. P. Home, D. Hanneke, J. D. Jost, D. Leibfried, and D. J. Wineland, Normal modes of trapped ions in the presence of anharmonic trap potentials, New J. Phys. 13, 073026 (2011).

[7] M. Mielenz, J. Brox, S. Kahra, G. Leschhorn, M. Albert, T. Schaetz, H. Landa, and B. Reznik, Trapping of topologicalstructural defects in Coulomb crystals, Phys. Rev. Lett. 110, 133004 (2013).

[8] B. Liu and J. Goree, Phonons in a one-dimensional Yukawa chain: Dusty plasma experiment and model, Phys. Rev. E 71, 046410 (2005).

[9] A. Melzer, Zigzag transition of finite dust clusters, Phys. Rev. E 73, 056404 (2006).

[10] T. E. Sheridan, Dusty plasma ring model, Phys. Scr. 80, 065502 (2009).

[11] T. E. Sheridan and K. D. Wells, Dimensional phase transition in small Yukawa clusters, Phys. Rev. E 81, 016404 (2010).

[12] T. E. Sheridan and A. L. Magyar, Power law behavior for the zigzag transition in a Yukawa cluster, Phys. Plasmas 17, 113703 (2010).

[13] A. V. Straube, R. P. A. Dullens, L. Schimansky-Geier, and A. A. Louis, Zigzag transitions and nonequilibrium pattern formation in colloidal chains, J. Chem. Phys. 139, 134908 (2013).

[14] J.-B. Delfau, C. Coste, and M. Saint Jean, Transverse singlefile-diffusion near the zigzag transition, Phys. Rev. E 87, 032163 (2013).

[15] J.-B. Delfau, C. Coste, and M. Saint Jean, Noisy zigzag transition, fluctuations, and thermal bifurcation threshold, Phys. Rev. E 87, 062135 (2013).

[16] T. Dessup, T. Maimbourg, C. Coste, and M. Saint Jean, Linear instability of a zigzag pattern, Phys. Rev. E 91, 022908 (2015).

[17] S. Fishman, G. De Chiara, T. Calarco, and G. Morigi, Structural phase transitions in low-dimensional ion crystals, Phys. Rev. B 77, 064111 (2008).

[18] G. De Chiara, A. del Campo, G. Morigi, M. B. Plenio, and A. Retzker, Spontaneous nucleation of structural defects in inhomogeneous ion chains, New J. Phys. 12, 115003 (2010).

[19] G. Piacente, G. Q. Hai, and F. M. Peeters, Continuous structural transitions in quasi-one-dimensional classical Wigner crystals, Phys. Rev. B 81, 024108 (2010).

[20] E. Shimshoni, G. Morigi, and S. Fishman, Quantum zigzag transition in ion chains, Phys. Rev. Lett. 106, 010401 (2011).
[21] J. E. Galvan-Moya and F. M. Peeters, Ginzburg-Landau theory of the zigzag transition in quasi-one-dimensional classical Wigner crystals, Phys. Rev. B 84, 134106 (2011).

[22] G. E. Astrakharchik, G. De Chiara, G. Morigi, and J. Boronat, Thermal and quantum fluctuations in chains of ultracold polar molecules, J. Phys. B. 42, 154026 (2009).

[23] P. Kolodner, D. Bensimon, and C. M. Surko, Travelling wave convection in an annulus, Phys. Rev. Lett. 60, 1723 (1988).

[24] D. Bensimon, B. I. Shraiman, and V. Croquette, Nonadiabatic effects in convection, Phys. Rev. A 38, 5461 (1988).

[25] J. J. Niemela, G. Ahlers, and D. S. Cannell, Localized traveling wave states in binary-fluid convection, Phys. Rev. Lett. 64, 1365 (1990).

[26] L. Bellon, L. Fourtune, V. T. Minassian, and M. Rabaud, Wavenumber selection and parity-breaking bifurcation in directional viscous fingering, Phys. Rev. E 58, 565 (1998).

[27] S. Douady, S. Fauve, and O. Thual, Oscillatory phase modulation of parametrically forced surface waves, Europhys. Lett. 10, 309 (1989).

[28] A. J. Simon, J. Bechhoefer, and A. Libchaber, Solitary modes and the Eckhaus instability in directional solidification, Phys. Rev. Lett. 61, 2574 (1988).

[29] G. Faivre, S. De Cheveigne, C. Guthmann, and P. Kurowski, Solitary tilt waves in thin lamellar eutectics. Europhys. Lett. 9, 779 (1989).

[30] M. Rabaud, S. Michalland, and Y. Couder, Dynamical regimes of directional viscous fingering: Spatiotemporal chaos and wave propagation, Phys. Rev. Lett. 64, 184 (1990).

[31] S. Fauve, S. Douady, and O. Thual, Comment on 'Paritybreaking transition of modulated patterns in hydrodynamic systems,' Phys. Rev. Lett. 65, 385 (1990).

[32] B. Caroli, C. Caroli, and S. Fauve, On the phenomenology of tilted domains in lamellar eutectic growth, J. Phys. I 2, 281 (1992).

[33] S. Fauve, Nonlinear dynamics of coupled oscillators, Ann. Phys. Fr. 19, 691 (1994).

[34] In an infinite system, $\phi(X, T)$ actually is a distance. In an annular or periodic system it is obviously mapped to an angle, thus to a phase.

[35] H. Goldstein, Classical Mechanics (Addison Wesley, Reading, MA, 1980).

[36] P. Coullet, R. E. Goldstein, and G. H. Gunaratne, Parity-breaking transition of modulated patterns in hydrodynamic systems, Phys. Rev. Lett. 63, 1954 (1989).

[37] P. Coullet and G. Iooss, Instabilities of one-dimensional cellular patterns, Phys. Rev. Lett. 64, 866 (1990).

[38] From Eqs. (2) and (3), we see that odd powers of $\epsilon^{1 / 2}$ appear in the power expansion of the distances as even derivatives of $\Phi$ and odd derivatives of $H$. In the expansion of the energy, the odd powers of $\epsilon^{1 / 2}$ necessarily include odd powers of such terms, and thus they change sign in a parity transformation. Since the resulting equations should be invariant under parity, odd powers of $\epsilon^{1 / 2}$ in the Lagrangian density should necessarily be in a factor of exact $X$ derivatives, as shown for the $\epsilon^{3 / 2}$ term in (5).

[39] E. Infeld and G. Rowlands, Nonlinear Waves, Solitons and Chaos (Cambridge University Press, Cambridge, 1990).

[40] To be more specific, let $\epsilon=\epsilon_{\text {wall }}\left(1-\delta^{2}\right)$ with $\delta \ll 1$. We obtain that $h(x) \approx d h_{b-}$, to first order in $\delta$, if $x \in[-d \ln \delta / \kappa, d \ln \delta / \kappa]$. 
[41] This justifies a posteriori the somewhat uncontrolled approximation in the estimation of the coefficient $g_{5}$.

[42] M. Abramowitz and I. A. Stegun, Handbook of Mathematical Functions (National Bureau of Standards, Washington, D.C., 1972).

[43] J.-B. Delfau, C. Coste, and M. Saint Jean, Single file diffusion of particles with long-ranged interactions: Damping and finite size effects, Phys. Rev. E 84, 011101 (2011).
[44] J.-B. Delfau, C. Coste, C. Even, and M. Saint Jean, Single-file diffusion of interacting particles in a finite-sized channel, Phys. Rev. E 82, 031201 (2010).

[45] I. S. Gradshteyn and I. M. Ryzhik, Table of Integrals, Series, and Products (Academic Press, San Diego, California, 2007).

[46] E. T. Whittacker and G. N. Watson, A Course of Modern Analysis (Cambridge University Press, Cambridge, 1920). 